\title{
Erratum to: Women and the Criminal Justice System
}

\author{
Emma Milne, Karen Brennan, Nigel South \\ and Jackie Turton
}

\section{Erratum to:}

\section{E. Milne et al. (eds.), Women and the Criminal Justice System, https://doi.org/10.1007/978-3-319-76774-1}

The original version of this book was inadvertently published with an outdated editor affiliation. The affiliation of the book editor, Emma Milne, has been corrected as follows:

Emma Milne

Middlesex University

London, UK

\footnotetext{
The updated online version of this book can be found at https://doi.org/10.1007/978-3-319-76774-1 